Journal of

Cognitive Neuroscience

\title{
Retinotopic and lateralized processing of spatial frequencies in human visual cortex during scene categorization
}

\begin{tabular}{|r|l|}
\hline Journal: & Journal of Cognitive Neuroscience \\
\hline Manuscript ID: & JOCN-2013-0041.R1 \\
\hline Manuscript Type: & Original \\
\hline Date Submitted by the Author: & n/a \\
\hline Complete List of Authors: & $\begin{array}{l}\text { Musel, Benoit; Laboratoire de Psychologie et NeuroCognition, Psychology } \\
\text { Bordier, Cécile; Grenoble Institut des Neurosciences, } \\
\text { Dojat, Michel; Grenoble Institut des Neurosciences, } \\
\text { Pichat, Cedric } \\
\text { Chokron, Sylvie; Unité Fonctionnelle Vision \& Cognition, Fondation } \\
\text { Ophtalmologique Rothschild } \\
\text { Le Bas, Jean-François; Plate-forme IRMaGe, CHU Grenoble } \\
\text { Peyrin, Carole; Laboratoire de Psychologie et NeuroCognition, Psychology }\end{array}$ \\
\hline Keywords: & $\begin{array}{l}\text { Hemispheric specialization, Perception: High-level vision, Functional MRI, } \\
\text { Occipital cortex }\end{array}$ \\
\hline
\end{tabular}




\title{
Retinotopic and lateralized processing of spatial frequencies in human visual cortex during scene categorization
}

\author{
Benoit Musel $^{1,2}$, Cécile Bordier ${ }^{3,4}$, Michel Dojat ${ }^{3,4}$, Cédric Pichat $^{1,2}$, Sylvie Chokron ${ }^{5,6}$, \\ Jean-François Le Bas ${ }^{7}$, and Carole Peyrin ${ }^{1,2, *}$ \\ ${ }^{1}$ CNRS UMR 5105, 38040 Grenoble, France \\ 2 Laboratoire de Psychologie et NeuroCognition, Université Pierre Mendès France, 38040 \\ Grenoble Cedex 9, France \\ ${ }^{3}$ INSERM, U836, BP 170, 38042 Grenoble Cedex 9, France \\ ${ }^{4}$ Grenoble Institut des Neurosciences, Université Joseph Fourier, BP 170, 38042 Grenoble \\ Cedex 9, France \\ ${ }^{5}$ Unité Fonctionnelle Vision \& Cognition, Fondation Ophtalmologique Rothschild, Paris, \\ France \\ ${ }^{6}$ Laboratoire de Psychologie de la Perception, CNRS UMR 8158, Université Paris Descartes, \\ Paris, France \\ ${ }^{7}$ Plate-forme IRMaGe, UJF - CHU - INSERM US 17 - CNRS UMS 3552, CHU Grenoble, CS \\ 10217, 38043 Grenoble Cedex 9, France \\ *Corresponding author: \\ Carole Peyrin, $\mathrm{PhD}$ \\ CNRS UMR 5105 \\ Université Pierre Mendès France, BP 47 \\ 38040 GrenobleCedex09 \\ France \\ carole.peyrin@upmf-grenoble.fr
}




\begin{abstract}
Using large natural scenes filtered in spatial frequencies, we aimed to demonstrate that spatial frequency processing could not only be retinotopically mapped, it could also be lateralized in both hemispheres. For this purpose participants performed a categorization task using large black and white photographs of natural scenes (indoors versus outdoors, with a visual angle of $24^{\circ} \times 18^{\circ}$ ) filtered in low spatial frequencies (LSF), high spatial frequencies (HSF), and nonfiltered scenes (NF), in block-designed fMRI recording sessions. At the group level, the comparison between the spatial frequency content of scenes revealed firstly that compared to HSF, LSF scene categorization elicited activation in the anterior half of the calcarine fissures linked to the peripheral visual field, whereas compared to LSF, HSF scene categorization elicited activation in the posterior part of the occipital lobes, which are linked to the fovea, according to the retinotopic property of visual areas. At the individual level, functional activations projected on retinotopic maps revealed that LSF processing was mapped in the anterior part of V1, whereas HSF processing was mapped in the posterior and ventral part of V2, V3 and V4. Moreover, at the group level, direct interhemispheric comparisons performed on the same fMRI data highlighted a right-sided occipito-temporal predominance for LSF processing, and a left-sided temporal cortex predominance for HSF processing, in accordance with hemispheric specialization theories. By using suitable method of analysis on the same data, our results enabled us to demonstrate for the first time that spatial frequencies processing is mapped retinotopically and lateralized in human occipital cortex.
\end{abstract}

Keywords: Natural scene; Hemispheric specialization; Retinotopy; Occipital cortex; Human vision. 


\section{Introduction}

It is widely agreed that the visual recognition of scenes is a fast, automatic and reliable process. Many studies attest to the importance of the Fourier components of images during scene processing. In terms of signal representation, an image can be expressed in the Fourier domain in terms of both amplitude and phase spectra (Field, 1987; Ginsburg, 1986; Hughes, Nozawa, \& Kitterle, 1996; Tolhurst, Tadmor, \& Chao, 1992). The amplitude spectrum highlights the dominant spatial scales (spatial frequencies) and dominant orientations of the image, and the phase spectrum describes the relationship between spatial frequencies. On the one hand, it is now well established that the primary visual cortex is mainly dominated by complex cells that respond preferentially to orientations and spatial frequencies (De Valois, Albrecht, \& Thorell, 1982a; De Valois, Yund, \& Hepler, 1982; Poggio, 1972; Shams \& von der Malsburg, 2002). On the other hand, simulation and psychophysical experiments have shown that information from low/medium frequencies of amplitude spectra is sufficient to allow scene categorization (Guyader, Chauvin, Peyrin, Herault, \& Marendaz, 2004; Torralba \& Oliva, 2003). These data support the influential models of visual recognition (Bar, 2003; Bullier, 2001; Hegdé, 2008; Peyrin et al., 2010; Schyns \& Oliva, 1994). According to these models, visual analysis starts with the parallel extraction of different elementary visual attributes at different spatial frequencies, in a predominantly "coarse-to-fine" processing sequence. The LSF in a scene, conveyed by fast magnocellular visual channels, might therefore activate visual pathways and subsequently access the occipital cortex and high-order areas in the dorsal stream (parietal and frontal) more rapidly than HSF, allowing an initial perceptual parsing of visual inputs, prior to their complete propagation along the ventral (inferotemporal) stream which ultimately mediates object recognition. This initial low-pass visual analysis might serve to refine the subsequent processing of HSF, conveyed more slowly by parvocellular visual channels to the ventral stream. The majority of visual models are based on spatial frequency processing, yet exactly how these are processed within the visual cortex remains unclear. In fact, the issue of retinotopic organization and/or the cerebral asymmetries for spatial frequency processing in the cortex is still a subject of debate in the literature. 
Firstly, many experimental arguments assume that specialization of spatial frequency processing is shared between the two hemispheres, with right hemispheric predominance for LSF processing and left hemispheric predominance for HSF processing. This hemispheric specialization has been observed either through behavioral studies on healthy subjects (Kitterle, Christman, \& Hellige, 1990; Peyrin, Chauvin, Chokron, \& Marendaz, 2003; Sergent, 1982) and neurological patients (Lamb, Robertson, \& Knight, 1990; Robertson \& Lamb, 1991; Robertson, Lamb, \& Knight, 1988), or through functional neuroimaging studies (Fink et al., 1996; Han et al., 2002; Heinze, Hinrichs, Scholz, Burchert, \& Mangun, 1998; Iidaka, Yamashita, Kashikura, \& Yonekura, 2004; Kenemans, Baas, Mangun, Lijffijt, \& Verbaten, 2000; Lux et al., 2004; Martinez, Di Russo, Anllo-Vento, \& Hillyard, 2001; Martinez et al., 1997; Yamaguchi, Yamagata, \& Kobayashi, 2000). However the hemispheric asymmetries in question were largely inferred from studies assessing hemispheric specialization in global and local processing. Using hierarchical visual stimuli consisting of a global form made up of several local elements (Navon, 1977), Sergent (1982) demonstrated a right hemispheric dominance for the recognition of global forms, and a left-sided dominance for the recognition of local forms (see also, Buchsbaum et al., 2006; Chokron, Brickman, Wei, \& Buchsbaum, 2000). Since global processing can be considered to be mediated by low-pass spatial analysis, and local processing by high-pass spatial analysis (Badcock, Whitworth, Badcock, \& Lovegrove, 1990; Lamb \& Yund, 1993; Shulman, Sullivan, Gish, \& Sakoda, 1986), the hemispheric specialization patterns observed in global and local processing have been interpreted as reflecting the hemispheric specialization for LSF and HSF, respectively (Iidaka, Yamashita, Kashikura, \& Yonekura, 2004; Peyrin, Baciu, Segebarth, \& Marendaz, 2004). Neuroimaging studies conducted on hierarchical visual stimuli have reported conflicting results on the cortical structures which present hemispheric specialization. For example, using Positron Emission Tomography, Fink et al. (1996) reported cerebral asymmetries in the occipital cortex. The right lingual gyrus was more highly activated during the processing of global as opposed to local forms, while the left inferior occipital gyrus was more highly activated during the processing of local rather than global forms. However, Heinze et al. (1998) failed to demonstrate cerebral asymmetries in first-stage visual areas. Instead, their results 
based on event-related potentials showed long latency asymmetries (260-360 latency range) for global versus local processing, suggesting that hemispheric specialization was only present in the higher levels of visual analysis. Thus, the imaging studies previously mentioned have provided conflicting results on hemispheric specialization using hierarchical stimuli. Using a method involving direct inter-hemispheric comparison, and by directly manipulating the spatial frequency content of stimuli, subsequent studies observed hemispheric specialization in certain occipito-temporal areas (Iidaka, Yamashita, Kashikura, \& Yonekura, 2004; Peyrin, Baciu, Segebarth, \& Marendaz, 2004). The direct inter-hemispheric comparison method examines contrasts between "unflipped" and "left-right flipped" functional images from the same experimental condition, in order to compare activity in one hemisphere with activity in homologous regions of the other hemisphere (Baciu, Juphard, Cousin, \& Le Bas, 2005; Cousin, Peyrin, \& Baciu, 2006; Iidaka, Yamashita, Kashikura, \& Yonekura, 2004; Lux et al., 2004; Peyrin, Baciu, Segebarth, \& Marendaz, 2004; Peyrin et al., 2005). Using this method in an event-related fMRI study, Peyrin et al. (2004) demonstrated greater activation in the right than in the left middle occipital gyrus during the recognition of LSF scenes, and greater activation in the left than in the right middle occipital gyrus during the recognition of HSF scenes. Importantly, when analysing the same fMRI data using a more traditional approach contrasting spatial frequencies to one another, these authors observed a stronger bilateral temporal activation for LSF than HSF scenes, while the reverse contrast did not reveal any significant activation. Thus, results differ according to the method of data analysis applied. The direct inter-hemispheric method of comparison seems more appropriate for the assessment of cerebral asymmetries, since it allows the cancelling out of any main effect deriving from spatial frequency bias.

Although a considerable number of studies postulate hemispheric specialization for spatial frequency processing, others highlight retinotopic processing for spatial frequencies in the occipital cortex. First of all, different imaging data obtained from patients with cerebral lesions (Holmes, 1918; Horton \& Hoyt, 1991) and from healthy participants (Engel, Glover, \& Wandell, 1997) show that the human primary visual cortex is retinotopically organized. The representation of the visual field ranges from posterior to anterior visual cortex, and shifts from 
the centre to the periphery. A large number of neurophysiological studies performed on cats (Everson et al., 1998; Issa, Trepel, \& Stryker, 2000), primates (De Valois, Albrecht, \& Thorell, 1982b; Foster, Gaska, Nagler, \& Pollen, 1985; Gegenfurtner, Kiper, \& Levitt, 1997; Tootell, Silverman, Hamilton, Switkes, \& De Valois, 1988; Xu, Anderson, \& Casagrande, 2007) and humans (Henriksson, Nurminen, Hyvärinen, \& Vanni, 2008; Sasaki et al., 2001; Singh, Smith, \& Greenlee, 2000) have mapped representations of the different spatial frequencies in retinotopic areas. Other studies have demonstrated that more complex cognitive functions, such as visual spatial attention, are also mapped consistently by cortical retinotopy (Brefczynski \& DeYoe, 1999; Gandhi, Heeger, \& Boynton, 1999; Martinez et al., 1999; Sasaki et al., 2001; Tootell et al., 1998; Watanabe et al., 1998). In particular, using retinotopic encoding with achromatic sinusoidal gratings, Sasaki et al. (2001) showed that low spatial frequencies were mapped according to the peripheral visual field, whereas high spatial frequencies were mapped according to the central visual field. Then, using very large hierarchical visual stimuli, these authors found evidence for retinotopic mapping of global and local attention in the occipital cortex. During "attend global" blocks, participants were required to deliberately focus their attention on the global form (at a visual angle of $29.4^{\circ}$ ) involving their peripheral vision, while during "attend local" blocks, they had to focus on the local elements (at a visual angle of $2.4^{\circ}$ ) involving more foveal vision. The results showed that when attention was directed at a local level, activation was consistent with the cortical representation of the fovea which was also sensitive to HSF gratings. When attention was directed at a global level, activation was consistent with the cortical representation of the periphery which was also sensitive to LSF gratings. Importantly, Sasaki et al. (2001) concluded that neither local nor global attention was lateralized in the occipital cortex. However, the authors used a traditional method of data analysis, comparing global and local experimental conditions to one another, rather than the direct inter-hemispheric comparison method used by Peyrin et al. (2004).

In the present fMRI study, we aimed to demonstrate that spatial frequency processing could not only be retinotopically mapped, it could also be lateralized in both hemispheres. We used a categorization task of natural scenes filtered in LSF and HSF, along with non-filtered (NF) scenes, in order to evaluate the retinotopy and cerebral asymmetries involved in spatial 
frequency processing. With this aim in mind, we used a block-design fMRI paradigm in which either large LSF, HSF or NF scenes were displayed in separate experimental blocks. We used large natural scenes (at a visual angle of $24^{\circ} \times 18^{\circ}$ ), covering as broad a visual field as in Sasaki et al. (2001). Large natural scenes have never been used previously to investigate the neural basis of spatial frequency processing within retinotopic areas. Natural scenes are more ecological and complex stimuli than those previously used (e.g., hierarchical stimuli, gratings) and allow an explicit change in the spatial frequency spectrum. Furthermore, natural scenes may be categorized in many frequency bands (i.e., whatever the type of filtering - low-pass, high-pass or pass-band). The retinotopic mapping of spatial frequency processing was assessed by projecting corresponding functional activation onto individual flat retinotpoic maps of each hemisphere where low level areas were delineated using a standard phase encoding experiments (Warnking et al., 2002). According to previous retinotopy studies, when processing spatial frequencies, the categorization of LSF scenes (relative to HSF) would recruit areas devoted to peripheral vision, whereas the categorization of HSF scenes (relative to LSF) would recruit areas devoted to foveal vision. Cerebral asymmetries were assessed using the direct inter-hemispheric method of comparison. Based on the results obtained by Peyrin et al. (2004), we expected a higher level of activation in right than in left occipito-temporal areas during the processing of LSF, and more involvement of the left hemisphere during HSF processing. The issue of retinotopic organization and/or the cerebral asymmetries for spatial frequency processing in the cortex is still a subject of debate in the literature and it has never been investigated previously on the same data. Our results demonstrate that spatial frequency processing is both retinotopically organized and lateralized in human visual cortex.

\section{Materials and Methods}

\subsection{Participants}

Eighteen right-handed participants (11 males; $25 \pm 3$ years) with normal or corrected-to-normal vision were scanned in this experiment. Participants with psychiatric or neurological disorders, or on medication were not included in the study. All participants gave their informed written consent before participating in the study, which was approved by the local ethics committee 
(CPP Sud Est V, France). All participants endorsed a spatial frequency processing experiment (Experiment 1) and for three of them an additional fMRI retinotopic mapping experiment (Experiment 2).

\subsection{Experiment 1: Spatial frequency processing}

\subsubsection{Stimuli and procedure}

Stimuli were 80 black and white photographs (256 grey scales), all with a visual angle of $24^{\circ} \mathrm{x}$ $18^{\circ}$, representing two categories: indoors and outdoors. They all had similar dominant orientations (as shown by the mean amplitude spectrum of non-filtered natural scenes in each category) to avoid their identification on the basis of this kind of cue (Guyader, Chauvin, Peyrin, Herault, \& Marendaz, 2004). For each scene, an LSF or an HSF stimulus was created (Figure 1). Filtered images were created using the image processing toolbox on MATLAB (Mathworks Inc., Sherborn, MA, USA). They were obtained by multiplying the Fourier transformation of the original images with Gaussian filters. We used a two-dimensional Gaussian centered on the null frequency with a maximal value equal to one, and standard deviations equal to 28 and 38 cycles per image. With this filter design we obtained a gaussian filter with a frequency cutoff (amplitude attenuation of square root of 1/2) of 1 cycle/degree of visual angle (i.e., low-pass cutoff of 24 cycles per image) for LSF stimuli, and below 1 cycle/degree (i.e., high-pass cutoff of 24 cycles per image) for HSF stimuli ${ }^{1}$. Then, the energy of LSF, HSF and non-filtered (NF) stimuli was equalized for each scene ${ }^{2}$. Each scene was presented in these three filtering conditions (NF, LSF and HSF) to each participant. Stimuli were displayed using E-prime software (E-prime Psychology Software Tools Inc., Pittsburgh, PA) and back-projected on a translucent screen positioned at the rear of the magnet. Participants viewed this screen at a distance of about $222 \mathrm{~cm}$ via a mirror fixed on the head coil.

A block-design paradigm with 6 types of stimuli (indoor scenes LSF, HSF, NF; outdoor scenes LSF, HSF, NF) was used. The experiment consisted of eight functional scans, and each functional scan was composed of 6 blocks (2 LSF-blocks, 2 HSF-blocks and 2 NF-blocks) including 10 images (5 indoors and 5 outdoors), and 2 blocks with a fixation dot in the center 
of the screen (fixation condition) displayed against a grey background. The order of blocks was randomized between scans. Each image was presented in the three filtering conditions, but each image did not appear more than once per scan. Each stimulus was displayed for $300 \mathrm{~ms}$ and the average interval between the onsets of two successive stimuli was $2.5 \mathrm{~s}$. The participants had to give a categorical answer ("indoors" or "outdoors") by pressing the corresponding key with the forefinger and the middle finger of their dominant hand. Half of the participants had to answer "indoors" with the forefinger and "outdoors" with the middle finger, while the second half of the participants had to answer "indoors" with the middle finger and "outdoors" with the forefinger. Response accuracy (ACC) and reaction times (RT, in milliseconds) were recorded.

\section{- Insert Figure 1 about here -}

\subsection{2. fMRI Acquisition}

A whole-body 3T MR scanner was used (Bruker MedSpec S300) with $40 \mathrm{mT} / \mathrm{m}$ gradient strength. For functional scans, the manufacturer-provided gradient-echo/T2* weighted EPI method was used. Thirty-nine adjacent axial slices parallel to the bi-commissural plane were acquired in interleaved mode. Slice thickness was $3.5 \mathrm{~mm}$. The in-plane voxel size was $3 \times 3$ $\mathrm{mm}(216 \times 216 \mathrm{~mm}$ field of view acquired with a $72 \times 72$ pixel data matrix; reconstructed with zero filling to $128 \times 128$ pixels). The main sequence parameters were: $\mathrm{TR}=2.5 \mathrm{~s}, \mathrm{TE}=30 \mathrm{~ms}$, flip angle $=77^{\circ}$. Acquisition time per functional run was $3 \mathrm{~min} 20 \mathrm{~s}$, allowing the acquisition of 80 volumes. To correct images for geometric distortions induced by local B0inhomogeneity, a B0 field map was obtained from two gradient echo data sets $(\Delta \mathrm{TE}=9.1 \mathrm{~ms})$ with the following common parameters: $\mathrm{TR}=25 \mathrm{~ms}$, acquisition matrix: $64 \times 256 \times 64$ $(\mathrm{LR} \times \mathrm{AP} \times \mathrm{CC})$, nominal resolution: $4 \times 1 \times 4 \mathrm{~mm}^{3}$. Slice orientation was set identical to the functional images. Finally, a T1-weighted high-resolution three-dimensional anatomical volume was acquired, by using a 3D Modified Driven Equilibrium Fourier Transform (MDEFT) sequence (field of view: $256 \times 224 \times 176 \mathrm{~mm}$; resolution: $1.333 \times 1.750 \times 1.375 \mathrm{~mm}$; acquisition matrix: $192 \times 128 \times 128$ pixels; reconstruction matrix: $256 \times 128 \times 128$ pixels). 


\subsubsection{Data analysis}

Data analysis was performed using the general linear model (Friston et al., 1995) for block designs in SPM8 (Wellcome Department of Imaging Neuroscience, London, UK, www.fil.ion. ucl.ac.uk/spm) implemented in MATLAB 7 (Mathworks Inc., Sherborn, MA, USA). Individual scans were realigned and uwarped, time-corrected, normalized to the MNI space and spatially smoothed by an 8-mm FWHM (Full Width at Half Maximum) Gaussian kernel. Times-series for each voxel were high-pass filtered $(1 / 128 \mathrm{~Hz}$ cutoff) to remove low-frequency noise and signal drift.

\subsubsection{Evaluation of cerebral correlates for spatial frequency processing}

Four conditions of interest (LSF, HSF, NF, and fixation) were modeled as four regressors convolved with a canonical hemodynamic response function. Movement parameters derived from realignment corrections ( 3 translations and 3 rotations) were also entered in the design matrix as additional factors of no interest. On an individual level, we firstly identified the brain regions involved in the processing of each spatial frequency content relative to the fixation ([LSF > fixation], [HSF > fixation] and [NF > fixation]). Then, we identified the neural correlates associated with each specific spatial frequency content by comparing the different filtering conditions to one another ([LSF $>$ NF], $[\mathrm{HSF}>\mathrm{NF}],[\mathrm{LSF}>\mathrm{HSF}]$ and $[\mathrm{HSF}>\mathrm{LSF}]$ ). Finally, two-stage random effect analyses were performed based on individual analyses using one sample t-tests. Clusters of activated voxels were then identified, based on the intensity of individual responses. Because the main effects of spatial frequency content (relative to fixation) were stronger than for between spatial frequency conditions, two different significance thresholds were applied. Therefore, for contrasts between spatial frequencies and the fixation periods, areas of activation were considered significant if they exceeded a voxel threshold of $\mathrm{p}<0.05 \mathrm{FWE}$ corrected for multiples comparisons, with a minimum cluster extent of 5 voxels. For contrasts between spatial frequency content, areas of activation were considered significant if they exceeded a less restrictive voxel threshold of $\mathrm{p}<0.001$ uncorrected $(\mathrm{T}>3.65)$, with a minimum cluster extent of 5 voxels. To facilitate comparisons 
with other studies, a transformation of MNI into Talairach and Tournoux (1988) coordinates was performed using the MNI2TAL function (created by Matthew Brett, available at http://www.mrc-cbu.cam.ac.uk/Imaging).

\subsubsection{Evaluation of hemispheric predominance in spatial frequency processing}

To assess hemispheric asymmetry in spatial frequency processing, we used a direct interhemispheric comparison method which compares the activity of both hemispheres (Baciu, Juphard, Cousin, \& Le Bas, 2005; Cousin, Peyrin, \& Baciu, 2006; Iidaka, Yamashita, Kashikura, \& Yonekura, 2004; Lux et al., 2004; Peyrin, Baciu, Segebarth, \& Marendaz, 2004; Peyrin et al., 2005). To avoid left-right morphological asymmetries, we normalized individual data using a symmetrical template built by averaging the EPI template available on SPM and its mirror around the midsagittal plane. Then, two sets of functional volumes were contrasted, one corresponding to the functional volumes of neurological convention (left is left) and the second set corresponding to the same functional volumes flipped $180^{\circ}$ in relation to the midsagittal plane (left is right), so that the second set represented "mirror" images of the first set. On an individual level, we first compared the unflipped and flipped activations obtained for the processing of each spatial frequency content [LSF_unflip > LSF_flip], [HSF_unflip > HSF_flip], and [NF_unflip > NF_flip]. In order to highlight cerebral activations directly linked to the hemispheric specialization for spatial frequency processing, we tested the interaction between spatial frequencies (LSF and HSF) and hemispheres (unflippled and flipped image). Then we compared the unflipped and flipped activations obtained for the processing of each spatial frequency content relative to fixation: [LSF-Fixation_unflip > LSF-Fixation_flip], [HSF-Fixation_unflip > HSF-Fixation_flip], and [NF-Fixation_unflip > NF-Fixation_flip]. Two-stage random effect analyses based on individual analyses were then performed using one sample t-tests. Clusters of activated voxels, based on the intensity of individual responses, were then identified, $(\mathrm{p}<0.001$ uncorrected, $\mathrm{T}>$ 3.65 , extent threshold of 5 voxels). While this uncorrected threshold may seem liberal, it is in line with those of our previous study reporting significant hemispheric differences within the occipital cortex (Peyrin et al., 2004). 


\subsection{Experiment 2: fMRI Retinotopic Mapping}

\subsubsection{Stimuli and procedure}

A ring slowly contracting or expanding about a fixation point (e.g., a central fixation cross) mapped eccentricity in the visual field. Speed of contraction or expansion varied linearly with eccentricity. Thus the activation wave on the cortical surface traveled at approximately constant speed (under the assumption of an exponential cortical magnification factor). When the ring reached maximum eccentricity (set at 3 degree) it wrapped around to be replaced by a new one at minimum eccentricity (set at 0.2 degree), and vice versa. Polar angle in the visual field was mapped by two wedges separated by a phase lag of 180 degree and rotating slowly at constant speed about the fixation point. Period of both eccentricity and polar angle stimulation was $32 \mathrm{~s}$. Stimulus parameters were the same as described in Warnking et al. (2002): rings and wedges consisted of a black and white radial checkerboard flickering at a frequency of $4 \mathrm{~Hz}$. Aspect ratio of the checks was kept constant by scaling their height linearly with eccentricity. Stimuli were created on MATLAB and displayed using homemade software written in $\mathrm{C}++$. The timing was controlled using the SDL library. Successive stimulus images were presented at a frequency of $4 \mathrm{~Hz}$, inducing the perception of an almost smoothly varying stimulus. Stimuli were started concomitantly with dummy MR excitations about $10 \mathrm{~s}$ prior to effective MR data acquisition so as to enable immediate response detection. To cancel out the effects of the hemodynamic delay, fMRI responses are compared to stimuli that cover the visual field in opposite direction - clockwise and counter-clockwise for polar angle encoded stimuli and expanded annuli and contracting annuli for the eccentricity encoded stimuli (Sereno, McDonald, \& Allman, 1994; Warnking et al., 2002). We acquired four retinotopic functional scans, one for each of the directions of motion of the rings and wedges.

Similarly to Experiment 1, stimuli were back-projected on a translucent screen positioned at the rear of the magnet. Participants viewed this screen at a distance of about $222 \mathrm{~cm}$ via a mirror fixed on the head coil. Observers were instructed to fixate a central fixation cross while paying attention to the whole stimulus. In order to maintain and control observers' attention they had to press a button each time the very small (just visible) fixation cross, displayed 
centrally, briefly changed of shape (form + to $\mathrm{x}$ and back to + ). However, we asked participants to try to span their spatial attention on the whole stimulus, as much as they could. During fMRI scanning, left eye position was monitored on-line by an infrared eye-tracker (ASL EyeTracker 6000, Applied Science Group). The eye-tracker was positioned on the floor behind the scanner and the translucent screen. A $3 \mathrm{~cm}$ radius hole was made at the bottom of the translucent screen so the camera monitored the participants's eye on the mirror and the infrared light emitter illuminated the pupil.

\subsection{2. fMRI Acquisition}

Functional, B0 field map and structural scans were acquired in a single scanning session on the same scanner as Experiment 1. For functional scans, thirty adjacent contiguous axial slices, angulated about the left-right axis to be approximately parallel to the calcarine sulcus, were acquired in interleaved mode. One of the central slices of the volume was positioned to contain as much of the calcarine sulcus as possible. Slice thickness was $3 \mathrm{~mm}$. The in-plane voxel size was $3 \times 3 \mathrm{~mm}(216 \times 192 \mathrm{~mm}$ field of view acquired with a $72 \times 64$ pixel data matrix $)$. The main sequence parameters were: $\mathrm{TR}=2 \mathrm{~s}, \mathrm{TE}=30 \mathrm{~ms}$, flip angle $=77^{\circ}$. The experiment consisted of four functional scans. Acquisition time per functional scan was $7 \mathrm{~min} 50 \mathrm{~s}$, allowing the acquisition of 235 volumes. The sequence parameters for the B0 field map scan were identical to Experiment 1. Finally, a high-resolution T1-weighted three-dimensional anatomical volume was acquired, by using a MP-RAGE sequence optimized based on (Deichmann, Good, Josephs, Ashburner, \& Turner, 2000). For each subject 176 sagittal partitions were acquired in two segments with an image matrix of $256 \times 112$ (read $\times$ phase). Further imaging sequence parameters were: $\mathrm{TR}=16 \mathrm{~ms}, \mathrm{TE}=4.96 \mathrm{~ms}, \mathrm{TI}=903 \mathrm{~ms}$, flip angle $=8^{\circ}$, fast phase encoding direction: AP (112 steps per RAGE train, 2 segments), slow phase encoding direction: LR, acquisition matrix: $256 \times 224 \times 176(\mathrm{CC} \times \mathrm{AP} \times \mathrm{LR})$, isotropic nominal resolution: $1 \mathrm{~mm}, \mathrm{BW}=$ $130 \mathrm{~Hz} / \mathrm{Px}$, readout direction: CC, number of averages: 1, total measurement time: $14 \mathrm{~min} 40 \mathrm{~s}$.

\subsubsection{Data analysis}

Assigning functional responses to a surface model of the cortex is particularly sensitive to 
geometric distortions of the 3D functional data due to static field inhomogeneity. Geometric distortions, if not corrected, really hamper the quality of the obtained retinotopic maps (Vasseur, Delon-Martin, Warnking, Segebarth, \& Dojat, 2010). Similarly to data from Experiment 1, the inhomogeneity of the static magnetic field was taken in to account during functional image preprocessing. A magnetic B0 field map was first calculated (Cusack \& Papadakis, 2002, Hutton et al., 2002) using the SPM8 Fieldmap Toolbox. The magnetic field map was further used to compute a voxel displacement map and then to correct all the functional images for the geometric distortions and to realign them with respect to the first one of the series. The conjoint field correction and realignment procedure was realized using the SPM8 Unwarp toolbox. In a final step, all EPI data sets were aligned with the structural data set using the SPM8 rigid body coregistration procedure.

\subsubsection{Model of the cortical surface}

Using a distributed Markovian method (Scherrer, Forbes, Garbay, \& Dojat, 2009), the structural volume was segmented into three tissue types: cerebro-spinal fluid, white matter and grey matter. After segmentation, the interface between volumes labeled as white matter and cortical grey matter was extended to represent approximately the center of the cortical surface. Some manual editing was performed to correct for topological defects detected in the occipital lobes. Then, the cortical surface situated in the occipital lobes was modeled. The modeled region was defined manually as a part of the whole segmented volume and extracted for each hemisphere. Within the delimited region, a triangulated model of the cortical surface interface was then generated following an approach (Scherrer et al., 2009) based on the marching cubes algorithm (Lorenson \& Cline, 1987). Distance between adjacent nodes of the triangulation was typically $1 \mathrm{~mm}$. Data were then analyzed on the folded, triangulated surface model. A flattened representation of the surface model was eventually generated for visualization purpose and used for automatic tracing of visual area borders.

\subsubsection{Retinotopic map generation}

The EPI datasets were analyzed voxel-wise by complex valued Fourier transformation, to 
determine amplitude and phase of the signals at the stimulation frequency (see details in Warnking et al., 2002). The results of the volume-based analysis of the (four) retinotopic scans were stored, for each visual coordinate, as a pair of parametric data volumes containing respectively phase and signal-to-noise ratio at the stimulation frequency. For generating retinotopic maps, phases were assigned to the cortical surface model as described in Warnking et al. (2002). The phase at each node of the surface model was estimated as a linear combination of the phases stored in a selection of voxels from the parametric data volume. Voxels from the parametric data volume that were retained in this selection had their center at most $3 \mathrm{~mm}$ away from the closest node of the surface model and a functional response at the stimulation frequency with a signal-to-noise ratio exceeding the value of 2. Following assignment of phase information to the surface model, retinotopic visual areas were automatically labeled and delineated on that model. The borders of the low order visual areas are determined from the detection of the changes of the visual field sign between adjacent areas (Sereno, 1994). To determine the visual field sign, it is then convenient to calculate the ratio ("visual field ratio" - VFR) of an oriented area measured using the local representation of the visual field coordinates with respect to some area measured using a locally isometric parameterization of the surface (this ratio is the Jaco-bian of the visual field representation on the surface). The visual area borders correspond to contour lines of zero VFR. Then, labeling retinotopic visual areas involves performing the following successive steps: 1. Application of a gaussian filter; 2. Calculation of a VFR map from the phase gradients with respect to a local two-dimensional coordinate system on the surface; 3 . Identification of candidates of the low order visual areas as those regions presenting a VFR and a signal-to-noise ratio of the smoothed eccentricity and polar angle phase maps beyond preset thresholds; 4. Selection of V1 as the largest among these candidates with negative VFR; and finally 5. Identification of the borders among retinotopic visual areas as the contour lines of zero VFR. Delineation of the visual areas from the VFR map was entirely performed in the two-dimensional Cartesian space of the flattened surface representation. In some cases, based on a visual inspection of the polar angle map, some manual editing may be introduced to refine the visual areas borders. 


\section{Results}

\subsection{Behavioral results}

Two $3 \times 2$ variance analyses (ANOVA) with Spatial frequency (LSF, HSF, and NF) and Categories (Indoors and Outdoors) as within-subjects factors were conducted on mean accuracy $(\mathrm{mACC})$ and mean reaction times $(\mathrm{mRT})$. On mACC, there was no effect of Spatial frequency $\left(F_{2,34}=1.36, p=.27\right)$ or of Category $\left(F_{1,17}<1\right)$. The data on mRT showed a main effect of Spatial frequency (LSF: $635 \pm 82 \mathrm{~ms}$, HSF: $620 \pm 86 \mathrm{~ms}$, NF: $611 \pm 78 \mathrm{~ms} ; F_{2,34}=$ $10.10, p<.01)$ but not of Category $\left(F_{1,17}=3.60, p=.08\right)$. Planned comparisons revealed that participants categorized HSF and NF more quickly than LSF conditions $\left(F_{1,17}=7.40, p<.05\right.$ and $F_{1,17}=22.52, p<.01$, respectively). We observed no difference between HSF scenes and NF scenes $\left(F_{1,17}=2.69, p=.11\right)$.

\subsection{Cerebral activation results}

\subsubsection{Retinotopic organization during spatial frequency processing}

By contrasting first the processing of natural scenes filtered in LSF, HSF and NF scenes to fixation blocks ([LSF $>$ fixation], [HSF $>$ fixation] and [NF > fixation] contrasts), we observed extensive recruitment of occipito-temporal areas, involving the bilateral lingual, fusiform and paraphippocampal gyri, and the right middle occipital gyrus for all contrasts. Then, the [LSF > HSF] contrast showed that LSF processing specifically activated the medial aspect of the occipital lobe, in the anterior half of the calcarine fissures (peak coordinates, $x, y, z: 0,-78,26$; Figure 2a and Table 1a). The reverse [HSF > LSF] contrast elicited significant rather more posterior bilateral activation in the cuneus (right hemisphere: $4 \mathrm{x},-88 \mathrm{y}, 15 \mathrm{z}$; left hemisphere: $9 \mathrm{x},-94 \mathrm{y}, 1 \mathrm{z}$ ), the lingual gyri and the right middle and superior occipital gyri (Figure $2 \mathrm{~b}$ and Table 1a). Regarding activation for scenes filtered in spatial frequencies compared to nonfiltered scenes, the $[\mathrm{LSF}>\mathrm{NF}]$ contrast and the $[\mathrm{HSF}>\mathrm{NF}]$ contrast presented no significant activated voxel $(\mathrm{p}<.001$ uncorrected). The opposite $[\mathrm{NF}>\mathrm{HSF}]$ and $[\mathrm{NF}>\mathrm{LSF}]$ contrasts elicited strong activation in the occipito-temporal cortex, with bilateral involvement of the cuneus, and the lingual, fusiform, and parahippocampal, gyri (Figure 2c-d and Table 1a). However, a higher level of anterior activation of the cuneus was noted for the [NF > HSF] 
$(15 x,-76 y, 9 z)$ than for the $[N F>L S F]$ contrast, and was very close to levels observed for the [LSF $>$ HSF] contrast. Additional activation was observed in the middle and superior occipital gyrus for the $[\mathrm{NF}>\mathrm{LSF}]$ contrast, and for the $[\mathrm{HSF}>\mathrm{LSF}]$ contrast.

We performed an additional group analysis in which we included reaction time as a covariate in order to remove cerebral activation due to reaction time differences across LSF and HSF processing. At the second level of the two-stage random effect analyses, we assigned as a covariate the mean reaction time of each spatial frequency condition to each participant. Here again, the [LSF > HSF] contrast showed that LSF processing specifically activated the medial aspect of the occipital lobe, in the anterior half of the calcarine fissures while the reverse $[\mathrm{HSF}>\mathrm{LSF}]$ contrast showed more posterior bilateral activation in the cuneus, the lingual gyri and the middle occipital gyri. Importantly, activations were very closed to those observed without reaction time covariate (see peak coordinates in Table 1a and 1b) suggesting that differences in reaction times across LSF and HSF scene categorization did not induce the retinotopic organization of cerebral activations during spatial frequency processing.

- Insert Figure 2 and Table 1 about here -

For three participants (P1, P2 and P3), we delineated retinotopic visual areas on the cortical surface of both hemispheres represented on Figure 3 as flat maps of the occipital volume, anteriorly delimitated by the parieto-occipital sulcus. Then, we projected their individual activations corresponding to spatial frequency processing ([LSF > HSF] and [HSF > HSF] contrasts; $\mathrm{p}<0.001$ uncorrected) in Experiment 1. Despite variability between participants, activation corresponding to HSF was located centrally and ventrally and activation corresponding to LSF was projected in periphery, outside the region covering $3^{\circ}$ (radius from the central fixation cross) of eccentricity in the visual field (see Figure 3). In some cases, LSF activation was more anterior and outside the occipital part of the brain that was unfolded.

For P1, the [LSF > HSF] contrast showed that LSF processing activated bilaterally the anterior part of V1, whereas the opposite [HSF $>$ LSF] contrast elicited activation in the posterior and 
ventral part of V2 and V3 of the left hemisphere. No relevant occipital activation was observed in the right hemisphere. For P2, the [LSF > HSF] contrast elicited bilateral activations in the anterior part of V1 and V2, extending to V3 in the right hemisphere, whereas the opposite $[\mathrm{HSF}>\mathrm{LSF}]$ contrast elicited bilateral activations in the posterior and ventral part of V2 and V3. For P3, the [LSF > HSF] contrast elicited bilateral activations in the anterior part of V1, whereas the opposite [HSF > LSF] contrast elicited bilateral activations in the posterior and ventral part of V2, V3 and V4 (these activations extending weakly to V1).

- Insert Figure 3 about here -

\subsubsection{Hemispheric predominance during spatial frequency processing}

To study hemispheric specialization during spatial frequency processing, we first compared both hemispheres for the LSF, HSF and NF blocks: [LSF_unflip > LSF_flip], [HSF_unflip > HSF_flip] and [NF_unflip > NF_flip] contrasts. Direct comparisons of the two hemispheres revealed which areas were more highly activated in one or the other in the same experimental conditions. However, all these contrasts showed significant activation in left motor areas and in the right cerebellum, in relation to the motor response given by the right-handed participants, but no significant activations within the occipito-temporal cortex. Furthermore, the LSF/HSF*unflip/flip interaction did not reveal significant activation. We hypothesized that the categorization of large spatial frequency scenes may involve several other low-level visual processes that could have attenuated or masked the cerebral asymmetries directly linked to the processing of spatial frequencies. The subtraction of the fixation condition to each spatial frequency condition (either unflipped or flipped) allow to remove low level visual processes and thus emphasize the activation linked to the categorization of spatial frequency scenes. Thus, we removed the fixation condition to each spatial frequency condition and compared both hemispheres for the LSF, HSF and NF blocks: [LSF-Fixation_unflip > LSF-Fixation_flip], [HSFFixation_unflip > HSF-Fixation_flip] and [NF-Fixation_unflip > NF-Fixation_flip] contrasts. All contrasts showed significant activation in left motor areas and in the right 
cerebellum, in relation to the motor response given by the right-handed participants, and in the right parahippocampal gyrus, the right cuneus and the left superior frontal gyrus. Critically, a hemispheric specialization for spatial frequency processing was observed in occipito-temporal areas (Figure 4 and Table 2). The [LSF-Fixation_unflip > LSF-Fixation_flip] contrast revealed that the right middle occipital and occipito-temporal gyri (BA 19/39, 39x, -76y, 15z and 48x, $70 \mathrm{y}, 15 \mathrm{z}$ ) were more highly activated than their left homologue during the processing of LSF. By contrast, the [HSF-Fixation_unflip > HSF-Fixation_flip] comparison revealed that the left middle temporal gyrus (BA 22, -59x $-33 y, 4 z)$ was more highly activated than its right homologue during the processing of HSF. The [NF-Fixation_unflip > NF-Fixation_flip] contrast revealed both that the middle occipito-temporal gyrus was more highly activated in the right than in the left hemisphere (BA 19/39, 48x, -70y, 15z), and that the middle temporal gyrus (BA 22, $-59 x,-42 y, 1 z)$ was more highly activated in the left than the right hemisphere.

- Insert Figure 4 and Table 2 about here -

\section{Discussion}

In the present block-design fMRI study, we used large natural scenes filtered in different spatial frequencies in order to ascertain which areas were involved in spatial frequency processing). We reported on eighteen participants extensive recruitment of occipito-temporal areas and found markers of hemispheric specialization within occipito-temporal areas. Furthermore, our results on three participants provided first of all evidence of a retinotopic organization of spatial frequency processing in human visual cortex.

First, we identified the cerebral correlates for spatial frequency processing irrespective of hemispheric dominance. The results of contrasts between filtering and fixation conditions ([LSF $>$ fixation], $[\mathrm{HSF}>$ fixation] and [NF $>$ fixation $]$ ) highlight the widespread recruitment of occipito-temporal areas, extending to the parahippocampal cortex, usually activated by scene stimuli. Similar parahippocampal activation during the perception of natural scenes had already been reported in a number of recent studies (Downing, Chan, Peelen, Dodds, \& Kanwisher, 2006; Epstein, 2008; Epstein, Harris, Stanley, \& Kanwisher, 1999; Epstein \& 
Kanwisher, 1998). More interestingly; by contrasting spatial frequency blocks to one another, we were able to show that the processing of spatial frequencies is related to the organization of retinotopic eccentricity in the occipital cortex. Activation related to the processing of LSF was observed in the medial occipital lobe, and in the anterior half of the calcarine fissure in relation to the peripheral representation, whereas activation linked to the processing of HSF was present in the posterior occipital lobes in relation to the foveal representation. These results are supported by the findings of Dougherty et al. (2003), who demonstrated that the representation of the fovea in human areas V1, V2 and V3 is centered on the lateral-ventral aspect of the occipital lobes at mean Talairach coordinates $-29 x,-78 y,-11$ and $25 x,-80 y,-9 z$, which is very close to the occipital activation observed for HSF (compared to LSF) scene categorization, particularly in the right hemisphere (peak Talairach coordinates: 27x, $-80 y,-3 z$ ). Our results could be explained by the distribution and neurophysiological properties of photoreceptor and ganglion cells in the human retina (Curcio \& Allen, 1990; Curcio, Sloan, Kalina, \& Hendrickson, 1990). First of all, the density of cones and midget ganglion cells, which are used to process HSF information, is greatest in the fovea. Since the fovea is represented in the posterior areas of the visual cortex, HSF information (conveyed by the parvocellular pathway to the visual cortex) might be predominantly processed in these areas. Moreover, the density of rods and parasol ganglion cells, which are used to process LSF information, increase with foveal eccentricity. Since the peripheral retina is represented in progressively more anterior areas of the visual cortex, LSF information (conveyed by the magnocellular pathway to the visual cortex) might be predominantly processed within these areas.

In addition to retinotopic activation, activation related to LSF processing was located in the upper (dorsal) part of the cuneus (BA 17), while HSF-related activation was located in the lower (ventral) part of the cuneus (BA 17/18), extending to the ventromedial cortex (at the level of the lingual gyrus). Because patients with achromatopsia have ventromedial occipital brain damage and attention to color activates this region (Bartels \& Zeki, 2000; Brewer, Liu, Wade, \& Wandell, 2002; Kaas, 1997; Wade, Brewer, Rieger, \& Wandell, 2005; Wandell, Brewer, \& Dougherty, 2005; Zeki et al., 1991), the cortex of the lingual gyrus has been suggested to be the human correlated of the monkey V4 of the ventral cortical stream. 
Therefore, our results suggest that HSF in scenes project on the occipital areas of the ventral stream. The activation observed could be linked with projections from the magno- and parvocellular pathways in the occipital areas of the dorsal and ventral visual streams, respectively (Goodale \& Milner, 1992; Mishkin, Ungerleider, \& Macko, 1983). Indeed, in monkeys, it has been shown that the magnocellular pathway project mostly to the occipital areas of the dorsal stream, beginning with the visual area V1, going through V2, then to V3 and the motion processing region MT (also known as V5) and to the posterior parietal cortex. The parvocellular pathway project to the occipital areas of the ventral stream, beginning with the visual area V1, going through $\mathrm{V} 2, \mathrm{~V} 3$ and then to the color processing region V4, and lastly to the complex form analysis regions in the inferior temporal cortex. In order to further explore this assumption, we overlapped the functional activations obtained in our scene categorization task with retinotopic maps acquired on three participants. For the three participants, the LSF processing activated mainly the anterior part of V1, whereas the HSF processing activated mainly the posterior part of the ventral regions of V2 (V2v) and V3 (V3v). Our results are consistent with the findings of Dougherty et al. (2003) who showed that the foveal representation is situated on the lateral-ventral regions of V1, V2 and V3. Importantly, to our knowledge, there is no neurophysiological evidence linking functional specialization along the lines of ventral and dorsal processing streams and the ventral and dorsal regions of V2 and V3. However, our results suggest that the HSF conveyed by the parvocellular pathway project mostly on the ventral regions of V2 and V3. Furthermore, for one participant (P3), HSF processing strongly activated V4, suggesting that HSF in scenes project on the occipital areas of the ventral stream. However, this assumption is based on only one participant's results. Furthermore, due to the individual variability the small subset of the population evaluated limits our conclusion. Additional experiments with more individual results will need to fully investigate the projection of spatial frequency processing on ventral and dorsal streams.

Our results are consistent with the retinotopic mapping of local and global attention orientation of hierarchical visual stimuli observed by Sasaki et al. (2001), and with the relationship between local and global information and spatial frequency processing (Badcock et al., 1990; 
Lamb \& Yund, 1993; Schulman et al., 1986). Attention directed to local level information preferentially activated the foveal representation of the cortex, where activation in response to HSF scene categorization is also highest. When attention is instead directed to global level, activation was consistent with the LSF scene categorization at more peripheral eccentricities. Our results were, however, different from those observed in Peyrin et al. (2004) who failed to observe retinotopic activation when comparing directly the neural correlates of LSF and HSF during the visual recognition of smaller scene images. Contrasting LSF to HSF scene recognition, significant activation was obtained in the right anterior temporal cortex and in the parahippocampal gyrus (reflecting semantic information and familiarity processes in scenes), as well as in the right inferior parietal lobule (reflecting attention-related processes), while no significant activation was observed by contrasting HSF and LSF scene recognition. The authors attributed these results to a whole LSF processing bias in their recognition task, which masked activation induced by HSF scene recognition. Indeed, in Peyrin et al. (2004), the highpass spatial frequency cutoff was higher than in the present study (over 6 cycles per degree of visual angle). That would result in a weaker visual signal and thus in a less pronounced BOLD signal for HSF than LSF scenes (below 4 cycles per degree of visual angle). To avoid an LSF processing bias in the present study, we used lower high- and low-pass spatial frequency cutoffs, that is over 1 cycle/degree of visual angle for HSF scenes and under 1 cycle/degree of visual angle for LSF scenes ${ }^{1}$.

Interestingly, activation observed when contrasting LSF and HSF blocks mirrors activation obtained when contrasting NF blocks and spatial frequency blocks. Indeed, the [NF > HSF] contrast in which activation from HSF scenes was subtracted from NF scenes indirectly highlight activation related to LSF processing, whereas the [NF > LSF] contrast in which activation from LSF scenes was subtracted from NF scenes indirectly highlight activation relating to HSF processing. Our results showed that activation of the anterior part of the cuneus induced by the $[\mathrm{NF}>\mathrm{HSF}$ ] was similar to the activation observed for the [LSF > HSF] contrast. In the same way, the more posterior and lateral activation of the cuneus observed for the $[\mathrm{NF}>\mathrm{LSF}]$ contrast is close to activation observed for the [HSF $>$ LSF] contrast. 
In addition to the retinotopic activation identified by contrasting spatial frequencies, we also demonstrated cerebral asymmetries for spatial frequency processing. In order to identify cerebral asymmetries (but also to remove any bias in spatial frequency processing when determining cerebral asymmetries), we directly compared the hemispheres by contrasting "unflip" to "left-right flip" functional images for each particular spatial frequency band (LSF, HSF and NF, relative to the fixation periods). First at all, the fMRI images were normalized using a symmetrical left-right template in order to avoid as possible as we could an effect of structural asymmetries between hemispheres when identifying the functional dissociation between homologous regions. Furthermore, it should be noted that these dissociations could not be due to differences in visual stimulations, because we compared hemispheres during the same visual events. Using this method, we found that spatial frequency processing was associated with differential hemispheric activation in occipito-temporal areas. As expected from previous studies (Iidaka, Yamashita, Kashikura, \& Yonekura, 2004; Peyrin, Baciu, Segebarth, \& Marendaz, 2004), the middle occipito-temporal junction (BA 19/39) was significantly and predominantly more highly activated in the right hemisphere during LSF scene categorization, whereas the anterior part of the middle temporal gyrus (BA 22) was significantly and predominantly more highly activated in the left hemisphere during HSF scene categorization. Again, the cerebral asymmetries highlighted during NF scene categorization mirrored those observed during LSF and HSF scene categorization. Indeed, as was the case for LSF scenes, activation of the middle occipital gyrus (BA 19) was predominantly more pronounced in the right hemisphere, and the middle temporal gyrus (BA 22) was predominantly more activated in the left hemisphere, as was the case for HSF scenes. The entire frequency spectrum of NF scenes involving both LSF and HSF emphasized occipitotemporal activation in the individual hemispheres. Furthermore, results revealed cerebral asymmetries irrespective of the spatial frequency content of scenes. Activation of the parahippocampal gyrus was greater in the right than in the left hemisphere. This result suggests that the parahippocampal gyrus activation observed when contrasting the spatial frequency content of individual scenes to the fixation was predominant in the right hemisphere. Activation of one part of the cuneus was also higher in the right than in the left hemisphere 
irrespective of spatial frequencies. An explanation for this unexpected response may be related to visuo-spatial constraints imposed by the large visual stimuli used. Our results suggest that the right cuneus may have to "work" more intensively to process visual information displayed in the left peripheral visual field, and this could be attributed to the superiority of the right hemisphere for the earlier perceptual level of processing (Grabowska \& Nowicka, 1996; Peyrin et al., 2006). Finally, superior frontal gyrus (BA 10) activation was greater in the left than in the right hemisphere. Previous studies of memory function have shown the involvement of frontal cortical area 10 in the intentional retrieval of non-verbal materiel (Cabeza \& Nyberg, 2000; Tulving, Markowitsch, Kapur, \& Habib, 1994). Activity in area 10 may be related to attempts to retrieve prototype information about scene exemplars, or to demands made on memory in order to find a successful match for the prototype. In the left hemisphere, this region might be preferentially associated with categorical visual spatial memory (e.g., one item is 'above' or 'below' the other; Slotnick \& Moo, 2006). In our study, categorical differences between indoor and outdoor scenes can be pinpointed in the visual organization of the elements which make up a scene (for example, outdoors, the ground is below the scene and the sky is above). Therefore, the predominant left superior frontal gyrus activation could be associated to the categorical memory processes involved in our scene categorization task.

Otherwise, our results were consistent with behavioral studies showing a left visual field/right hemisphere predominance during the recognition of LSF scenes and a right visual field/left hemisphere predominance during HSF scene recognition (Peyrin, Chauvin, Chokron, \& Marendaz, 2003; Peyrin, Mermillod, Chokron, \& Marendaz, 2006). However, on a neurobiological level, the pattern of cerebral asymmetries is not strictly the same as the one reported by Peyrin et al. (2004). As far as LSF scene categorization is concerned, the right predominance in the middle occipital gyrus is consistent with the one observed in Peyrin et al. (2004), but also in numerous neuroimaging studies (Fink et al., 1996; Han et al., 2002; Iidaka, Yamashita, Kashikura, \& Yonekura, 2004; Lux et al., 2004). However, in our study, the HSF scene categorization elicited a left-sided predominance in the middle temporal cortex, which differed from the usual left occipital cortex predominance observed in previous neuroimaging studies. This result is more consistent with those obtained on neurological patients, with lesions 
centered in the left posterior superior temporal gyrus, showing a specific impairment of local element processing in hierarchical visual form (Lamb, Robertson, \& Knight, 1990; Robertson, Lamb, \& Knight, 1988). The discrepancy between our results and those of Peyrin et al. (2004) could be due to the high-pass spatial frequency cutoff of scenes. As previously mentioned, we excluded in the present study all spatial frequency content below 1 cycle/degree of visual angle for HSF (while 6 cycles/degree in Peyrin et al., 2004) in order to avoid a LSF processing bias when directly comparing LSF and HSF. The additional medium-range spatial frequencies in HSF scenes could explain the HSF advantage in reaction times. Alternatively, this HSF advantage might be attributed to the long presentation time of scenes (300 ms) favoring the categorization of scenes on the basis of HSF information (Peyrin, Chauvin, Chokron, \& Marendaz, 2003; Schyns \& Oliva, 1994). However, we do not consider this experimental manipulation to be detrimental to our goals, since Christman, Kitterle, and Hellige (1991) have previously shown that hemispheric asymmetries for spatial frequency processing are relative rather than absolute. The actual downside of this manipulation is that our HSF scenes also contained medium-range spatial frequencies (between 1 and 6 cycles/degree). Consequently both left and right occipital cortices might be involved in the categorization of our HSF scenes, thus masking the left occipital predominance previously observed in Peyrin et al. (2004), and in this case cerebral asymmetries may occur only in higher level areas of the ventral visual stream in the temporal regions (Tanaka, 1993). It should be noted that the behavioral HSF advantage in reaction times might be also interpreted as an effect of the right-hand used by the participants. Indeed, the right hand could preactivate the left hemisphere and thus facilitate the processing of HSF (relative to LSF) for which this hemisphere is specialized. In conclusion, using stimuli filtered in spatial frequencies that covered a large part of the visual field, and suitable methods of data analysis, we have succeeded in showing that the processing of spatial frequencies is retinotopically organized and lateralized in human visual cortex.

\section{Acknowledgment}

This work was supported by Région Rhone-Alpes. The authors warmly thank the "Délégation à la Recherche Clinique et à l'Innovation" of the University Hospital of Grenoble for sponsoring. 
We thank the technical staff at the $3 \mathrm{~T}$ scanner facility (Laurent Lamalle and Irène Troprès) from the UMS iRMaGe and Catherine Dal Molin for the English revision of the manuscript. 


\section{Footnotes}

${ }^{1}$ These particular spatial frequency cutoffs were selected based on our previous works. First, we aimed to avoid a bias of a spatial frequency band. Indeed, in Peyrin et al. (2004), spatial frequency cutoffs were below 4 cycle/degree of visual angle for LSF stimuli and above 6 cycles/degree for HSF stimuli. The [HSF > LSF] contrast did not reveal significant activation, suggesting a LSF bias. In a subsequent work not published, spatial frequency cutoffs were lower for LSF (below 1 cycle/degree of visual angle) and above 6 cycles/degree for HSF stimuli. Again, the [HSF > LSF] contrast did not reveal significant activation. Both studies indicated to use lower spatial frequency cutoff for HSF stimuli. In our parallel works conducted on patient with age-related macular degeneration (AMD) to demonstrate a specific HSF deficit in AMD, 1 cycle/degree was used as the cutoff frequency for HSF filtering. Therefore, in order to maintain consistency with this work and to keep the objective in mind of assessing AMD patients with the present fMRI paradigm, we selected 1 cycle/degree as the cutoff frequency for LSF and HSF filtering.

${ }^{2}$ The energy of a signal is equal to the sum of the squared values of the signal. The energy of LSF and HSF stimuli was equalized for each scene in the spatial domain as follow: $\operatorname{If} \operatorname{LSF}(i, j)$ and $\operatorname{HSF}(i, j)$ represent the value of the pixel at position $(i, j)$ of respectively the low and the high-pass filtered images of a scene, their energies are given by $E_{L S F}=\sum_{i, j} L S F(i, j)^{2}$ and $E_{H S F}=\sum_{i, j} H S F(i, j)^{2}$, respectively. The stimuli are then normalized by a fixed energy $E$, $\operatorname{LSF}_{\text {norm }}(i, j)=\operatorname{LSF}(i, j) \cdot E / E_{L S F}$ and $H S F_{\text {norm }}(i, j)=H S F(i, j) \cdot E / E_{H S F}$. It should be noted that this method of normalization was preferred to a RMS contrast normalization since our preliminary works showed that RMS contrast normalization drastically increases activation for HSF scenes only, such as no significant activation was obtained for the [LSF $>$ HSF] contrast, irrespective of the spatial frequency cutoff.

${ }^{3}$ All participants have to categorize scenes only with their dominant right hand in order to ensure the reliability of our inter-hemispheric comparison method. Indeed, using the right hand, the inter-hemispheric comparison should highlight a left side motor predominance whatever the spatial frequency particular band of natural scenes. The [LSF-Fixation_unflip > LSF-Fixation_flip], [HSF-Fixation_unflip > HSF-Fixation_flip] and [NF-Fixation_unflip > 
NF-Fixation_flip] contrasts showed indeed significant activation in left motor areas and in the right cerebellum, in relation to the motor response given by the right-handed participants.

1

2

3

4

5

6

7

8

9

10

11

12

13

14

15

16

17

18

19

20

21

22

23

24

25

26

27

28

29

30

31

32

33

34

35

36

37

38

39

40

41

42

43

44

45

46

47

48

49

50

51

52

53

54

55

56

57

58

59

60 


\section{References}

Baciu, M., Juphard, A., Cousin, E., \& Le Bas, J. F. (2005). Evaluating fMRI methods for assessing hemispheric language dominance in healthy subjects. European Journal of Radiology, 55(2), 209-218.

Badcock, J. C., Whitworth, F. A., Badcock, D. R., \& Lovegrove, W. J. (1990). Low-frequency filtering and the processing of local-global stimuli. Perception, 19(5), 617-629.

Bar, M. (2003). A cortical mechanism for triggering top-down facilitation in visual object recognition. Journal of Cognitive Neuroscience, 15(4), 600-609.

Bartels, A., \& Zeki, S. (2000) The architecture of the colour centre in the human visual brain: new results and a review. European Journal of Neuroscience, 12,172-193.

Brefczynski, J. A., \& DeYoe, E. A. (1999). A physiological correlate of the 'spotlight'of visual attention. Nature Neuroscience, 2, 370-374.

Brewer, A. A., Liu, J., Wade, A. R., \& Wandell, B. A. (2005). Visual field maps and stimulus selectivity in human ventral occipital cortex. Nature Neuroscience, 8(8),1102-1109.

Buchsbaum, M. S., Buchsbaum, B. R., Chokron, S., Tang, C., Wei, T. C., \& Byne, W. (2006). Thalamocortical circuits: fMRI assessment of the pulvinar and medial dorsal nucleus in normal volunteers. Neuroscience Letters, 404(3), 282-287.

Bullier, J. (2001). Integrated model of visual processing. Brain Research Reviews, 36(2-3), 96107.

Cabeza, R., \& Nyberg, L. (2000). Imaging cognition II: An empirical review of 275 PET and fMRI studies. Journal of Cognitive Neuroscience, 12(1), 1-47.

Chokron, S., Brickman, A. M., Wei, T., \& Buchsbaum, M. S. (2000). Hemispheric asymmetry for selective attention. Cognitive Brain Research, 9(1), 85-90.

Cousin, E., Peyrin, C., \& Baciu, M. (2006). Hemispheric predominance assessment of phonology and semantics: A divided visual Weld experiment. Brain and Cognition, 61, 298-304.

Curcio, C. A., \& Allen, K. A. (1990). Topography of ganglion cells in human retina. The Journal of Comparative Neurology, 300(1), 5-25.

Curcio, C. A., Sloan, K. R., Kalina, R. E., \& Hendrickson, A. E. (1990). Human photoreceptor topography. The Journal of Comparative Neurology, 292, 497-523.

Cusack, R., \& Papadakis, N. (2002). New robust 3-D phase unwrapping algorithms: application to magnetic field mapping and undistorting echoplanar images. NeuroImage, 16, 754-764.

Deichmann, R., Good, C. D., Josephs, O., Ashburner, J., \& Turner, R. (2000). Optimization of 3-D MP-RAGE sequences for structural brain imaging. NeuroImage, 12(1), 112-127

De Valois, R. L., Albrecht, D. G., \& Thorell, L. G. (1982a). Spatial frequency selectivity of cells in macaque visual cortex. Vision Research, 22, 545-559.

De Valois, R. L., Albrecht, D. G., \& Thorell, L. G. (1982b). Spatial frequency selectivity of cells in macaque visual cortex. Vision Research, 22(5), 545-559.

De Valois, R. L., Yund, E. W., \& Hepler, N. (1982). The orientation and direction selectivity of cells in macaque visual cortex. Vision Research, 22(5), 531-544.

Dougherty, R. F., Koch, V. M., Brewer, A. A., Fischer, B., Modersitzki, J., \& Wandell, B. A. (2003). Visual field representations and locations of visual areas $V 1 / 2 / 3$ in human visual cortex. Journal of Vision, 3(10), 586-598.

Downing, P. E., Chan, A. W. Y., Peelen, M. V., Dodds, C. M., \& Kanwisher, N. (2006). Domain specificity in visual cortex. Cerebral Cortex, 16(10), 1453-1461.

Engel, S. A., Glover, G. H., \& Wandell, B. A. (1997). Retinotopic organization in human visual cortex and the spatial precision of functional MRI. Cerebral Cortex, 7(2), 181192. 
Epstein, R. A. (2008). Parahippocampal and retrosplenial contributions to human spatial navigation. Trends in Cognitive Sciences, 12(10), 388-396.

Epstein, R. A., Harris, A., Stanley, D., \& Kanwisher, N. (1999). The Parahippocampal Place Area:: Recognition, Navigation, or Encoding? Neuron, 23(1), 115-125.

Epstein, R. A., \& Kanwisher, N. (1998). A cortical representation of the local visual environment. Nature, 392(6676), 598-601.

Everson, R. M., Prashanth, A. K., Gabbay, M., Knight, B. W., Sirovich, L., \& Kaplan, E. (1998). Representation of spatial frequency and orientation in the visual cortex. Proceedings of the National Academy of Sciences, 95(14), 8334-8338.

Field, D. J. (1987). Relations between the statistics of natural images and the response properties of cortical cells. Journal of the Optical Society of America A, 4(12), 23792394.

Fink, G. R., Halligan, P. W., Marshall, J. C., Frith, C. D., Frackowiak, R. S. J., \& Dolan, R. J. (1996). Where in the brain does visual attention select the forest and the trees?, Nature, $382,626-628$.

Foster, K. H., Gaska, J. P., Nagler, M., \& Pollen, D. A. (1985). Spatial and temporal frequency selectivity of neurones in visual cortical areas V1 and V2 of the macaque monkey. The Journal of Physiology, 365(1), 331-363.

Friston, K. J., Holmes, A. P., Worsley, K. J., Poline, J. B., Frith, C. D., \& Frackowiak, R. S. J. (1995). Statistical parametric maps in functional imaging: a general linear approach. Human Brain Mapping, 2(4), 189-210.

Gandhi, S. P., Heeger, D. J., \& Boynton, G. M. (1999). Spatial attention affects brain activity in human primary visual cortex. Proceedings of the National Academy of Sciences, 96(6), 3314-3319.

Gegenfurtner, K. R., Kiper, D. C., \& Levitt, J. B. (1997). Functional properties of neurons in macaque area V3. Journal of Neurophysiology, 77(4), 1906-1923.

Ginsburg, A. P. (1986). Spatial filtering and visual form perception. In K. Boff, L. Kauman \& J. Thomas (Eds.), Hanbook of perception and human performance (Vol. II, chap.34, pp. 1-41). New York: Wiley.

Goodale, M. A., \& Milner, A. D. (1992). Separate visual pathways for perception and action. Trends in Neurosciences, 15(1), 20-25.

Grabowska, A., \& Nowicka, A. (1996). Visual-spatial-frequency model of cerebral asymmetry: A critical survey of behavioral and electrophysiological studies. Psychological Bulletin, 120(3), 434-449.

Guyader, N., Chauvin, A., Peyrin, C., Herault, J., \& Marendaz, C. (2004). Image phase or amplitude? Rapid scene categorization is an amplitude-based process. Comptes Rendus Biologies, 327(4), 313-318.

Han, S., Weaver, J. A., Murray, S. O., Kang, X., Yund, E. W., \& Woods, D. L. (2002). Hemispheric asymmetry in global/local processing: Effects of stimulus position and spatial frequency. NeuroImage, 17(3), 1290-1299.

Hegdé, J. (2008). Time course of visual perception: Coarse-to-fine processing and beyond. Progress in Neurobiology, 84(4), 405-439.

Heinze, H. J., Hinrichs, H., Scholz, M., Burchert, W., \& Mangun, G. R. (1998). Neural mechanisms of global and local processing: A combined PET and ERP study. Journal of Cognitive Neuroscience, 10(4), 485-498.

Henriksson, L., Nurminen, L., Hyvärinen, A., \& Vanni, S. (2008). Spatial frequency tuning in human retinotopic visual areas. Journal of Vision, 8(10), 1-13.

Holmes, G. (1918). Disturbances of vision by cerebral lesions. British Journal of Ophthalmology, 2(7), 353-384.

Horton, J. C., \& Hoyt, W. F. (1991). The representation of the visual field in human striate cortex: a revision of the classic Holmes map. Archives of Ophthalmology, 109(6), 816. 
Hughes, H. C., Nozawa, G., \& Kitterle, F. L. (1996). Global precedence, spatial frequency channels, and the statistic of the natural image. Journal of Cognitive Neuroscience, 8 , 197-230.

Hutton, C., Bork, A., Josephs, O., Deichmann, R., Ashburner, J., \& Turner, R. (2002). Image distortion correction in fMRI: A quantitative evaluation. NeuroImage, 16(1), 217-240.

Iidaka, T., Yamashita, K., Kashikura, K., \& Yonekura, Y. (2004). Spatial frequency of visual image modulates neural responses in the temporo-occipital lobe. An investigation with event-related fMRI. Cognitive Brain Research, 18(2), 196-204.

Issa, N. P., Trepel, C., \& Stryker, M. P. (2000). Spatial frequency maps in cat visual cortex. The Journal of Neuroscience, 20(22), 8504-8514.

Kaas, J.H. (1997). Theories of visual cortex organization in primates. In J.H. Kaas, K.S. Rockland \& A. Peters (Eds), Cerebral cortex (pp. 91-125). New York: Plenum Press.

Kenemans, J. L., Baas, J. M. P., Mangun, G. R., Lijffijt, M., \& Verbaten, M. N. (2000). On the processing of spatial frequencies as revealed by evoked-potential source modeling. Clinical Neurophysiology, 111(6), 1113-1123.

Kitterle, F. L., Christman, S., \& Hellige, J. B. (1990). Hemispheric differences are found in the identification, but not the detection, of low versus high spatial frequencies. Attention, Perception, \& Psychophysics, 48(4), 297-306.

Lamb, M. R., Robertson, L. C., \& Knight, R. T. (1990). Component mechanisms underlying the processing of hierarchically organized patterns: Inferences from patients with unilateral cortical lesions. Journal of Experimental Psychology: Learning, Memory, and Cognition, 16(3), 471-483.

Lamb, M. R., \& Yund, E. W. (1993). The role of spatial frequency in the processing of hierarchically organized stimuli. Attention, Perception, \& Psychophysics, 54(6), 773 784.

Lorenson, W., \& Cline, H. (1987). Marching cubes, a high resolution 3D surface construction algorithm. Computer Graphics, 21(4), 163-169.

Lux, S., Marshall, J. C., Ritzl, A., Weiss, P. H., Pietrzyk, U., Shah, N. J., et al. (2004). A functional magnetic resonance imaging study of local/global processing with stimulus presentation in the peripheral visual hemifields. Neuroscience, 124(1), 113-120.

Martinez, A., Anllo-Vento, L., Sereno, M. I., Frank, L. R., Buxton, R. B., Dubowitz, D. J., et al. (1999). Involvement of striate and extrastriate visual cortical areas in spatial attention. Nature Neuroscience, 2, 364-369.

Martinez, A., Di Russo, F., Anllo-Vento, L., \& Hillyard, S. A. (2001). Electrophysiological analysis of cortical mechanisms of selective attention to high and low spatial frequencies. Clinical Neurophysiology, 112(11), 1980-1998.

Martinez, A., Moses, P., Frank, L., Buxton, R., Wong, E., \& Stiles, J. (1997). Hemispheric asymmetries in global and local processing: evidence from fMRI. NeuroReport, 8(7), 1685-1689.

Mishkin, M., Ungerleider, L. G., \& Macko, K. A. (1983). Object vision and spatial vision: Two cortical pathways. Trends in Neurosciences, 6, 414-417.

Musel, B., Hera, R., Chokron, S., Alleysson, D., Chiquet, C., Romanet, J.P., Guyader, N., \& Peyrin, C. (2011). Residual abilities in age-related macular degeneration patients to process spatial frequencies during natural scenes categorization. Visual Neuroscience, 28, 529-541.

Navon, D. (1977). Forest before trees: The precedence of global features in visual perception. Cognitive Psychology, 9(3), 353-383.

Peyrin, C., Baciu, M., Segebarth, C., \& Marendaz, C. (2004). Cerebral regions and hemispheric specialization for processing spatial frequencies during natural scene recognition, an event-related fMRI study. NeuroImage, 23(2), 698-707. 
Peyrin, C., Chauvin, A., Chokron, S., \& Marendaz, C. (2003). Hemispheric specialization for spatial frequency processing in the analysis of natural scenes. Brain and Cognition, 53(2), 278-282.

Peyrin, C., Chokron, S., Guyader, N., Gout, O., Moret, J., \& Marendaz, C. (2006). Neural correlates of spatial frequency processing: A neuropsychological approach. Brain Research, 74, 1-10.

Peyrin, C., Mermillod, M., Chokron, S., \& Marendaz, C. (2006). Effect of temporal constraints on hemispheric asymmetries during spatial frequency processing. Brain and Cognition, 62(3), 214-220.

Peyrin, C., Michel, C. M., Schwartz, S., Thut, G., Seghier, M., Landis, T., et al. (2010). The neural substrates and timing of top-down processes during coarse-to-fine categorization of visual scenes: a combined fMRI and ERP study. Journal of Cognitive Neuroscience, $22,2768-2780$.

Peyrin, C., Schwartz, S., Seghier, M., Michel, C., Landis, T., \& Vuilleumier, P. (2005). Hemispheric specialization of human inferior temporal cortex during coarse-to-fine and fine-to-coarse analysis of natural visual scenes. NeuroImage, 28(2), 464-473.

Poggio, G. F. (1972). Spatial properties of neurons in striate cortex of unanesthetized macaque monkey. Investigative Ophthalmology \& Visual Science, 11(5), 368-377.

Robertson, L. C., \& Lamb, M. R. (1991). Neuropsychological contributions to theories of part/whole organization. Cognitive Psychology, 23(2), 299-330.

Robertson, L. C., Lamb, M. R., \& Knight, R. T. (1988). Effects of lesions of temporal-parietal junction on perceptual and attentional processing in humans. The Journal of Neuroscience, 8(10), 3757-3769.

Sasaki, Y., Hadjikhani, N., Fischl, B., Liu, A. K., Marret, S., Dale, A. M., et al. (2001). Local and global attention are mapped retinotopically in human occipital cortex. Proceedings of the National Academy of Sciences of the United States of America, 98(4), 2077-2082.

Sereno, M. I., McDonald, C. T., \& Allman, J. M. (1994). Analysis of retinotopic maps in extrastriate cortex. Cerebral Cortex, 4, 601-620.

Scherrer, B., Forbes, F., Garbay, C., \& Dojat, M. (2009). Distributed local MRF models for tissue and structure brain segmentation. IEEE Transactions on Medical Imaging, 28(8), 1278-1295.

Schyns, P. G., \& Oliva, A. (1994). From blobs to boundary edges: Evidence for time- and spatial-scale-dependant scene recognition. Psychological Science, 5(4), 195-200.

Sergent, J. (1982). The cerebral balance of power: Confrontation or cooperation? Journal of Experimental Psychology: Human Perception and Performance, 8(2), 253-272.

Shams, L., \& von der Malsburg, C. (2002). The role of complex cells in object recognition. Vision Research, 42(22), 2547-2554.

Shulman, G. L., Sullivan, M. A., Gish, K., \& Sakoda, W. J. (1986). The role of spatialfrequency channels in the perception of local and global structure. Perception, 15(3), 259-273.

Singh, K. D., Smith, A. T., \& Greenlee, M. W. (2000). Spatiotemporal frequency and direction sensitivities of human visual areas measured using fMRI. NeuroImage, 12(5), 550-564.

Slotnick, S. D., \& Moo, L. R. (2006). Prefrontal cortex hemispheric specialization for categorical and coordinate visual spatial memory. Neuropsychologia, 44(9), 1560-1568.

Talairach, J., \& Tournoux, P. (1988). Co-planar stereotaxic atlas of the human brain: 3dimensional proportional system: an approach to cerebral imaging. New York: Thieme.

Tanaka, K. (1993). Neuronal mechanisms of object recognition. Science, 262(5134), 685.

Tolhurst, D. J., Tadmor, Y., \& Chao, T. (1992). Amplitude spectra of natural images. Ophthalmic and Physiological Optics, 12(2), 229-232. 
Tootell, R. B. H., Hadjikhani, N., Hall, E. K., Marrett, S., Vanduffel, W., Vaughan, J. T., et al. (1998). The retinotopy of visual spatial attention. Neuron, 21(6), 1409-1422.

Tootell, R. B. H., Silverman, M. S., Hamilton, S. L., Switkes, E., \& De Valois, R. L. (1988). Functional anatomy of macaque striate cortex. V. Spatial frequency. The Journal of Neuroscience, 8(5), 1610-1624.

Torralba, A., \& Oliva, A. (2003). Statistics of natural image categories. Network: Computation in Neural Systems, 14(3), 391-412.

Tulving, E., Markowitsch, H. J., Kapur, S., \& Habib, R. (1994). Novelty encoding networks in the human brain: Positron emission tomography data. NeuroReport, 5, 2525-2528.

Vasseur, F., Delon-Martin, C., Warnking, J., Segebarth, C., \& Dojat, M. (2010). fMRI retinotopic mapping at 3T: Benefits gained from correcting the spatial distortions due to static field inhomogeneity. Journal of Vision, 10(12), 1-12.

Wade, A. R., Brewer, A. A., Rieger, J. W., \& Wandell, B. A. (2002) Functional measurements of human ventral occipital cortex: retinotopy and color. Philosophical Transactions of the Royal Society of London. Series B, Biologicial Sciences, 357(1424), 963-973.

Wandell, B. A., Brewer, A. A., \& Dougherty, R. F. (2005). Visual field map clusters in human cortex. Philosophical Transactions of the Royal Society of London. Series B, Biologicial Sciences, 360(1456), 693-707.

Warnking, J., Dojat, M., Guérin-Dugué, A. Delon-Martin, C., Olympieff, S., Richard, N. et al. (2002). fMRI retinotopic mapping - step by step. NeuroImage, 17(4),1665-1683.

Watanabe, T., Sasaki, Y., Miyauchi, S., Putz, B., Fujimaki, N., Nielsen, M., et al. (1998). Attention-regulated activity in human primary visual cortex. Journal of Neurophysiology, 79(4), 2218-2221.

$\mathrm{Xu}, \mathrm{X}$., Anderson, T. J., \& Casagrande, V. A. (2007). How do functional maps in primary visual cortex vary with eccentricity? The Journal of Comparative Neurology, 501(5), 741-755.

Yamaguchi, S., Yamagata, S., \& Kobayashi, S. (2000). Cerebral asymmetry of the" top-down" allocation of attention to global and local features. The Journal of Neuroscience, 20(9), RC72.

Zeki, S., Watson, J. D., Lueck, C. J., Friston, K. J., Kennard, C., Frackowiak, R. S. (1991). A direct demonstration of functional specialization in human visual cortex. The Journal of Neuroscience, 11(3), 641-649. 


\section{Figure Captions}

Figure 1. Example of stimuli used in the four experimental conditions: low spatial frequencies (LSF), high spatial frequencies (HSF), non-filtered (NF) scenes and Fixation periods.

Figure 2. Activated regions obtained by contrasting (a) [LSF > HSF], (b) [HSF > LSF], (c) $[\mathrm{NF}>\mathrm{HSF}]$, and (d) [NF $>\mathrm{LSF}]$.

Figure 3. Individual activation for three participants (P1, P2 and P3). The central row represents individual activation maps elicited by the $[\mathrm{LSF}>\mathrm{HSF}]$ and $[\mathrm{HSF}>\mathrm{LSF}]$ contrasts $(\mathrm{p}<0.001$ uncorrected) in Experiment 1. Individual activations were projected on the corresponding left (left row) and right (right row) flat hemispheres for each participant. On each flat map, retinotopic areas were delineated based on the retinotopic mapping experiment. We indicated for each participant and each hemisphere, the activation corresponding to eccentricity mapping. The color legend represents a circular area of the visual field with an eccentricity of $3^{\circ}$ (radius from the central fixation cross). The black line represents the $3^{\circ}$ (radius) of eccentricity in each visual field. Only the occipital lobe was unfolded, anteriorly delimitated by the parieto-occipital sulcus. Ventral part and dorsal part of the 3D volume are respectively down and up on the unfolded surface.

Figure 4. Activated regions obtained using a direct inter-hemispheric comparison for low spatial frequency scenes (LSF; [LSF-Fixation_unflip > LSF-Fixation_flip] contrast), high spatial frequency scenes (HSF; [HSF-Fixation_unflip > HSF-Fixation_flip] contrast, and nonfiltered scenes (NF; [NF-Fixation_unflip > NF-Fixation_flip] contrast). The activation from the group analysis is projected onto a symmetrical template brain by averaging the T1 template available on SPM and its mirror around the midsagittal plane. The bottom of the figure shows the superposition of all activations. All contrasts showed activation in left motor areas [1], the right parahippocampal gyrus [2] and the right cuneus [3]. A hemispheric specialization for spatial frequency processing was observed in occipito-temporal areas (indicated by arrows in the figure), involving the right middle occipital gyrus [4] for LSF (as well as NF) and the left middle temporal gyrus [5] for HSF (as well as NF). 
Figure 1. Example of stimuli used in the four experimental conditions: low spatial frequencies (LSF), high spatial frequencies (HSF), non-filtered (NF) scenes and Fixation periods. $40 \times 19 \mathrm{~mm}(300 \times 300 \mathrm{DPI})$ 


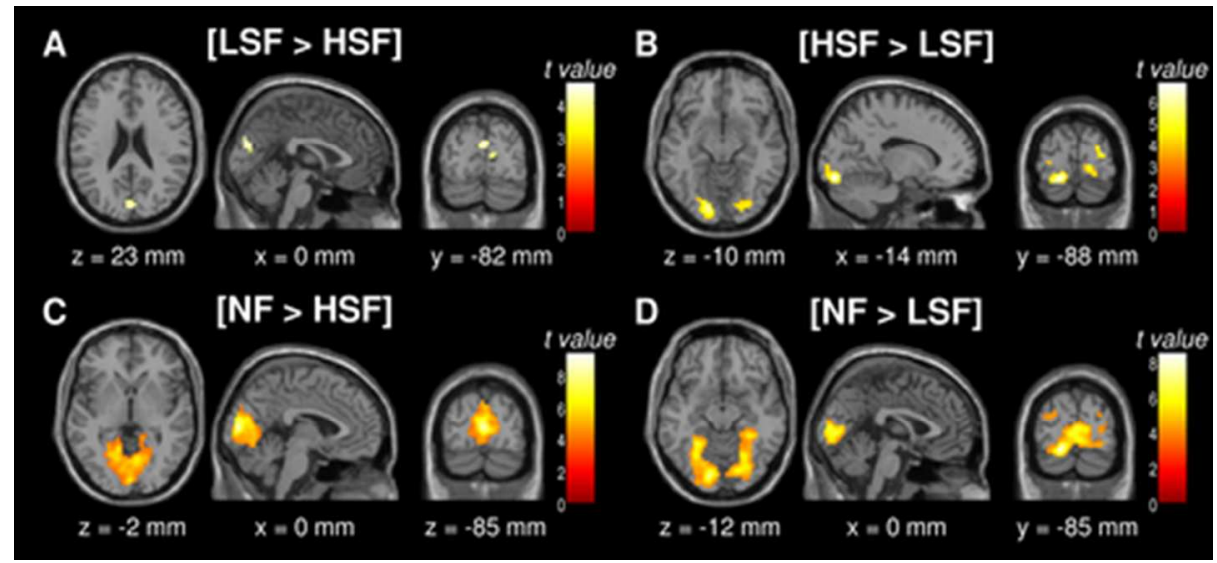

Figure 2. Activated regions obtained by contrasting (a) [LSF > HSF], (b) $[\mathrm{HSF}>\mathrm{LSF}]$, (c) $[\mathrm{NF}>\mathrm{HSF}]$, and (d) $[\mathrm{NF}>\mathrm{LSF}]$. $38 \times 17 \mathrm{~mm}(300 \times 300$ DPI $)$ 


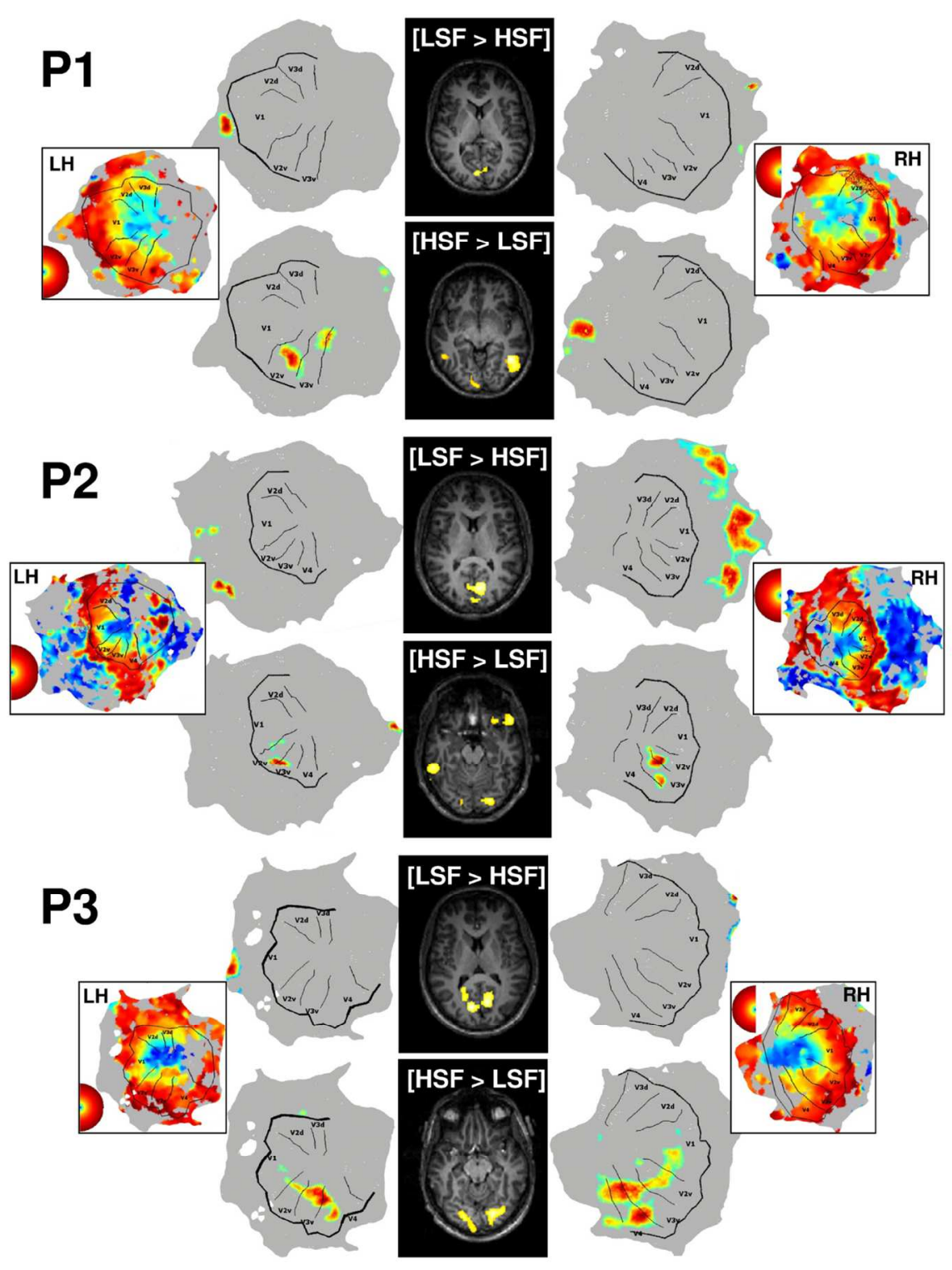

Figure 3. Individual activation for three participants (P1, $P 2$ and $P 3)$. The central row represents individual activation maps elicited by the [LSF > HSF] and [HSF > LSF] contrasts $(p<0.001$ uncorrected) in Experiment 1. Individual activations were projected on the corresponding left (left row) and right (right row) flat hemispheres for each participant. On each flat map, retinotopic areas were delineated based on the retinotopic mapping experiment. We indicated for each participant and each hemisphere, the activation corresponding to eccentricity mapping. The color legend represents a circular area of the visual field with an eccentricity of $3^{\circ}$ (radius from the central fixation cross). The black line represents the $3^{\circ}$ (radius) of eccentricity in each visual field. Only the occipital lobe was unfolded, anteriorly delimitated by the parietooccipital sulcus. Ventral part and dorsal part of the 3D volume are respectively down and up on the unfolded surface. $114 \times 156 \mathrm{~mm}(300 \times 300 \mathrm{DPI})$ 


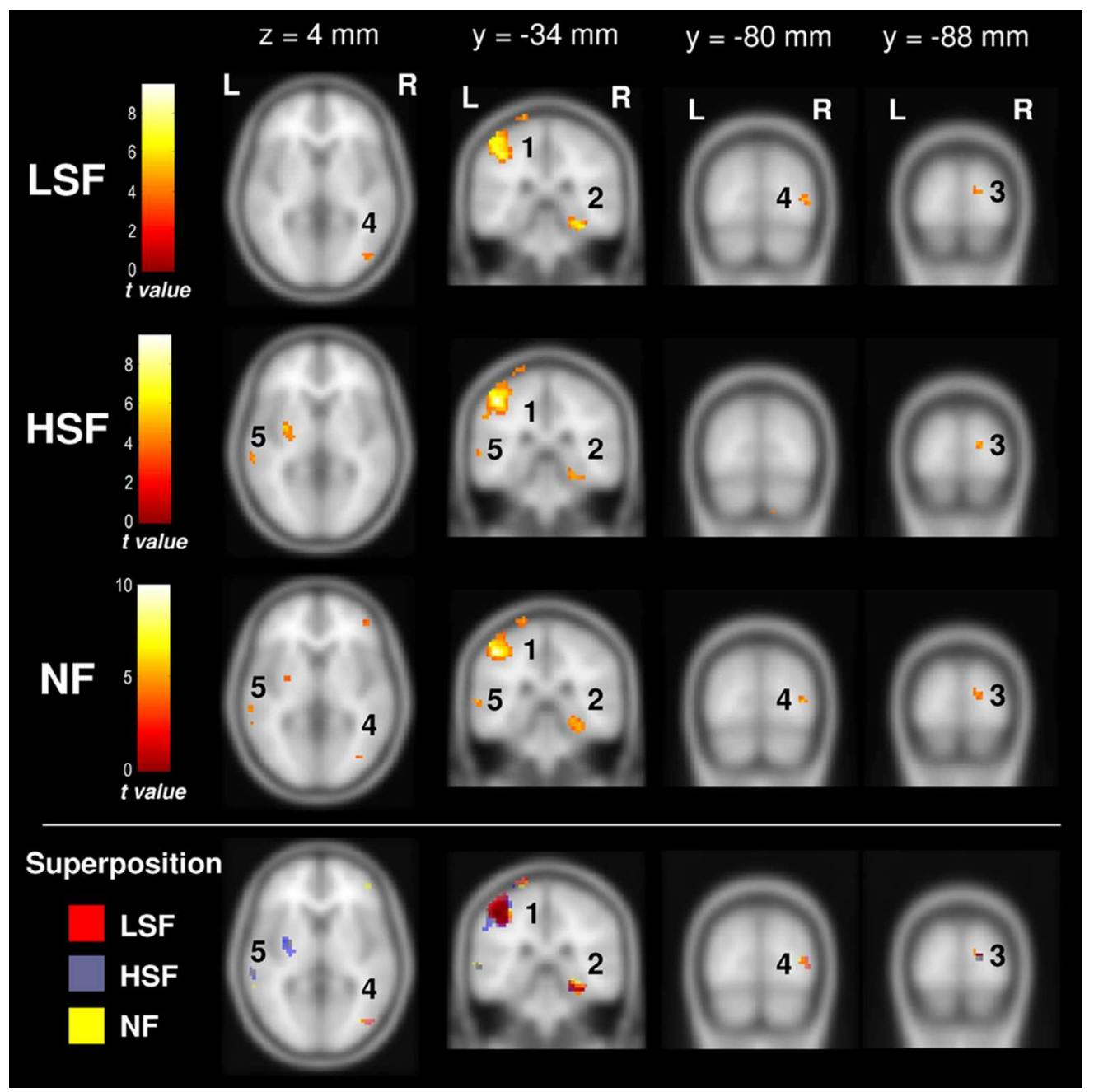

Figure 4. Activated regions obtained using a direct inter-hemispheric comparison for low spatial frequency scenes (LSF; [LSF-Fixation_unflip > LSF-Fixation_flip] contrast), high spatial frequency scenes (HSF; [HSF-

Fixation_unflip > HSF-Fixation_flip] contrast, and non-filtered scenes (NF; [NF-Fixation_unflip > NF-

Fixation_flip] contrast). The activation from the group analysis is projected onto a symmetrical template brain by averaging the T1 template available on SPM and its mirror around the midsagittal plane. The bottom of the figure shows the superposition of all activations. All contrasts showed activation in left motor areas [1], the right parahippocampal gyrus [2] and the right cuneus [3]. A hemispheric specialization for spatial frequency processing was observed in occipito-temporal areas (indicated by arrows in the figure), involving the right middle occipital gyrus [4] for LSF (as well as NF) and the left middle temporal gyrus [5] for HSF (as well as NF). $85 \times 85 \mathrm{~mm}(300 \times 300$ DPI $)$ 